\title{
CLUSTER ANALYSIS TO DETECT PATTERNS OF DRUG USE FROM ROUTINELY COLLECTED MEDICAL DATA
}

\author{
Sara Khalid, M. Sanni Ali, Peter Rijnbeek, Daniel Prieto-Alhambra
}

\begin{abstract}
Appropriate drug prescription for an increasingly ageing and multi-morbid population can be a challenge for general practitioners. This study uses unsupervised learning methods to identify different types of patient profiles which could inform policymakers and regulators about patterns of drug use, and identify specific clusters of users with unknown drug effects (risk and benefit). Hard and soft clustering methods are proposed to detect patterns of medication use by patients and to estimate the probability of belonging to a certain patient profile. Results showed the presence of expected as well as a surprising patient profile based on fracture risk factors. Challenges associated with unsupervised learning using electronic medical record data are described and an approach for evaluating models in the presence of unlabeled data using internal and external cluster evaluation methods is presented, such that it can be extended to other unsupervised learning applications within healthcare and beyond. To our knowledge, this is the first study proposing cluster analysis for detecting drug utilisation patters from electronic healthcare records in the routinely-collected SIDIAP database.
\end{abstract}

Keywords-unsupervised learning, electronic health records, cluster evaluation

\section{INTRODUCTION}

\section{A. The Clinical Context}

Drug utilization incurs significant costs to healthcare systems and is associated with adverse drug reactions resulting in $11 \%$ (all ages) and 5-6\% (over 65s) of hospital admissions [1]. For an increasingly ageing and frail population, this includes use of anti-fracture/ anti-osteoporosis drugs (AOD) [2]. Since most guidelines are based on clinical trials, ensuring "appropriate" prescription and use of medication in real practice conditions is difficult. Hence regulators are faced with uncertainty when medications are approved and then used by groups of patients seldom explored in pivotal (pre-marketing) trials.

There is therefore a need for data on the effectiveness and safety of long-term preventive drugs such as AODs in actual practice conditions. This is particularly relevant for certain subgroups of patients who, although potentially at high fracture risk, were under-represented or actively excluded in RCTs, such as users of oral glucocorticoids, males, cognitively impaired, octogenarians, patients with type 2 diabetes, kidney failure, or obesity. Similarly, the benefit-risk of preventive drugs might be suboptimal in patients at low risk of the event to be prevented (e.g. fracture in relation to AOD use).

* Research supported by the National Osteoporosis Society.

Both Authors are with the Centre for Statistics in Medicine, Nuffield Department of Orthopaedics Rheumatology and Musculoskeletal Sciences,

\section{B. Our Approach}

In this paper we propose an approach for detecting sub-groups within a population of AOD users by clustering together patients with similar fracture risk factors related to AOD use. Our aim is to 1) understand patterns of drug use, and 2) define patient clusters pertaining to under-studied population groups, to ultimately inform regulatory decision making.

We demonstrate the complexities involved with applying unsupervised methods to electronic health records data, and use both internal and external evaluation methods to assess the clustering solution. In addition to characterising clusters, the degree of a patient's membership in multiple clusters is also estimated. Although natural language processing methods have been used for mining electronic health records for drug safety studies [3, 4], this is to our knowledge the first study using cluster analysis to detect drug utilisation patterns in the SIDIAP database.

\section{Methods}

\section{A. Data}

The SIDIAP database (www.sidiap.com) contains anonymised electronic medical records of universally provided primary care for over $80 \%$ of the population of Catalonia, Spain [5]. A total of 145,236 records were extracted for the years 2012-2016.

In the literature, risk factors associated with osteoporosis include age, gender, body mass index, smoking, alcohol drinking, Charlson index (a measure of co-morbidities), steroid and sedative use, and fracture history. Expert opinion was used to confirm these as the main factors under consideration when prescribing medication for osteoporosis in primary care. Patients with missing data on any of these variables were excluded. Table 1 summarises the characteristics of the remaining 37,996 patients included in the study. All characteristics except for age, body mass index, and Charlson index occur as binary variables in the database. For this study we also categorised age, body mass index, and Charlson index since for visualization of the clustering solution, clustering variables must all be of the same data type.

An initial assessment of the data revealed no strong correlations (Pearson's correlation $>0.6$ ) between any of the variables, A Hopkin's statistic [6] of 0.44 was found suggesting that the data appear to be randomly distributed

University of Oxford, UK, OX3 7LD (e-mail sara.khalid@ndorms.ox.ac.uk) 


\begin{tabular}{|l|c|c|}
\hline \multicolumn{1}{|c|}{ Feature } & Description & Prevalence \\
\hline Elderly & Age $\geq 60$ years & $83.3 \%$ \\
\hline Female & Female gender & 79.4 \\
\hline Obese & BMI $\geq 30$ & 33.6 \\
\hline Smoker & Current use $^{\mathrm{a}}$ & 7.7 \\
\hline Drinker & Current use $^{\mathrm{a}}$ & 1.1 \\
\hline Comorbid & $\begin{array}{c}\text { Charlson index }^{-1} \\
\text { Current use }\end{array}$ & 52.1 \\
\hline Steroid user & $\begin{array}{c}\text { Current use } \\
\text { a }\end{array}$ & 43.3 \\
\hline Sedative user & $\begin{array}{c}\text { Any major } \\
\text { fracture (including } \\
\text { hip and non-hip) }\end{array}$ & 18.5 \\
\hline $\begin{array}{l}\text { Previously } \\
\text { fractured }\end{array}$ & \multicolumn{1}{|c|}{} \\
\hline
\end{tabular}

Table 1 Prevalence of features in the dataset.

a. As opposed to previous use or never used.

(in data space) rather than clustered into any obvious grouping structure.

\section{B. Cluster Analysis}

We begin with the hypothesis that there are sub-groups or clusters within the population of AOD users. An underlying assumption is that there is an association between the clustering pattern and the risk factors related to AOD use (shown in Table 1). In unsupervised learning, the "true" number of such clusters is not known. Therefore a candidate number of clusters $(k)$ may be sought based, e.g., on expert knowledge.

Our task then is to learn the structure of these $k$ clusters. There are several approaches for cluster analysis [7]. Among the most popular ones are $k$-means [8], and hierarchical clustering [9]. These methods provide "hard" cluster membership i.e. a subject can belong to exactly one cluster. Other methods, e.g. Gaussian mixture models (GMM) [10] and fuzzy c-means clustering perform "soft" clustering, i.e. where the subject can belong to more than one clusters according to a degree of membership. GMM is not suitable for binary data and are not considered here.

\section{Hierarchical clustering}

Let the $i$-th subject be represented in $d$-dimensional space data space by $x_{i}$, where $i=1: n$, and $d$ is the number of features. Each subject is initially considered to be a cluster. Based on a measure of similarity (e.g. Euclidean distance in $d$ dimensional space), the closest clusters are merged. Merging continues until all subjects have been merged into either the pre-specified number of clusters $(k)$, or one cluster ${ }^{1}$.

\section{$k$-means}

$k$ randomly selected points in the $d$-dimensional space are initially assigned to be cluster centroids. Each subject is allocated to the closest centroid (based on e.g. Euclidean distance). Cluster centroids are re-calculated based on the allocations. Subject re-allocation and centroid re-positioning continues until the location of cluster centroids is fixed.

\section{Fuzzy c-means clustering}

As with $k$-means, the algorithm seeks to find optimal cluster centroids. However, instead of assigning a given subject to exactly one cluster, the degree of membership of the subject within $k$ clusters is calculated, such that $\sum_{j=1}^{k} p\left(j \mid x_{i}\right)=1$, where $p\left(j \mid x_{i}\right)$ is the probability of membership in the $j$-th cluster for the given subject $x_{i}$ [11]. This allows subjects to belong to more than one clusters.

The clustering solution for both $k$-means and fuzzy $c$-means depends on the initialisation of cluster centroids. Therefore it is important to perform the clustering with several random initialisations.

\section{Cluster Evaluation}

\section{Internal}

Cluster evaluation assesses how well the clustered data fit within the candidate $k$ clusters, by estimating how well a clustering solution maximises homogeneity within a cluster and separation between clusters. The number of clusters for which a given evaluation criterion is met is considered to be the optimal number of clusters, $\hat{k}$.

$\hat{k}$ was determined by applying hierarchical and $k$-means clustering. From the clinical perspective, it was of interest to detect up to 10 clusters. For $k=2$ to 10 , clustering was performed 100 times, using a random sample of 1000 subjects in each iteration. Two distance metrics were used: squared Euclidean and city-block [ref]. Evaluation criteria used (described in literature) were: $\mathrm{CH}$, gap, and silhouette.

\section{External}

Once $k$ clusters were detected, we characterised the clusters further by considering information from the electronic medical records not included in the clustering process. Data on bone mineral density (with \% missingness) (BMD) and incident hip fracture risk (FR) were available. BMD and FR of $k$ clusters were examined and (where possible) compared to general population average [12] to get a measure of positioning of groups relative to the general population.

\section{RESUlts}

Figure 1 shows the cluster evaluation results for $k$-means and hierarchical clustering methods. In general, higher values of $\mathrm{CH}$, gap, and silhouette indicate better within-cluster homogeneity and between-cluster separation. It may be seen that for both $k$-means and hierarchical clustering, $\mathrm{CH}$ decreases from $k=2$ onward, suggesting $\hat{k}=2$. On the other hand, according to the gap criteria, using either $k$-means or hierarchical, $k=2$ results in the smallest gap and further there is no clear elbow point, as $k$ increases, where the gap is

\footnotetext{
${ }^{1}$ This is agglomerative clustering. Alternatively, divisive clustering may be performed where instead of merging, the dataset is iteratively divided into clusters.
} 
maximised. The silhouette criteria shows similar results to gap for $k$-means (indicating better clustering as $k$ increases).

However for hierarchical clustering it suggests that the clustering solution initially becomes worse as $k$ increases from 2 to 4 , but then from $k=5$ the clustering solution stabilizes somewhat and then worsens again at $k=8$. These results are based on Euclidean distance. Similar results (not shown) were obtained using city-block metric.
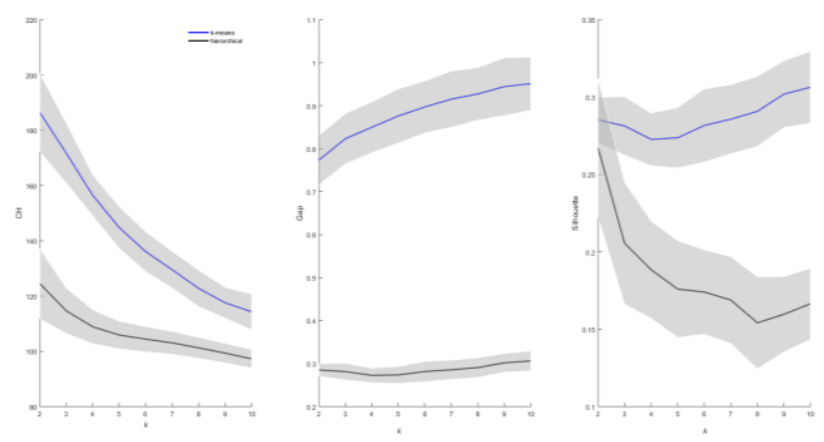

Figure 1. Internal evaluation of hierarchical (black) and $k$-means (blue) clustering solutions using the $\mathrm{CH}$ (Calinski-Harabasz) (left), gap (centre), and silhouette (right) criteria. Error bars (gray) show over 100 iterations.

Figure 1 is informative since it demonstrates that a) hierarchical clustering and $k$-means do not necessarily agree in their clustering solution, and b) there is no clear elbow point and stable state for the evaluation curves. Therefore error search methods which are typically employed to determine $\widehat{k}$ (see [13] for details in search methods) would not be appropriate to use here. Based on this, we cannot really say that there is an obvious optimal number of clusters in our data.
This highlights an important complexity of cluster analysis when applied to real world medical data in that determination of an optimal grouping distribution is not straightforward.

Figure 2 shows a two-dimensional view of the distribution of 5 fuzzy clusters. For ease of visualization, two of the continuous variables (age and BMI) are selected for a twodimensional plot. As expected the cluster distributions overlap and each subject in the dataset takes membership of more than one cluster.

Next we examine the clusters detected by hierarchical clustering using $k=2,3,4$, and 5 (Figure 3). At $k=2$ the two clusters that are detected differ in gender. One has $99.9 \%$ males and the other is all females. As $k$ increases, the male cluster remains intact, while the female cluster is further divided. At $k=3$, the female cluster is divided into a group of $100 \%$ elderly and non-smokers and another group containing $25 \%$ elderly, $50 \%$ smokers, $86 \%$ women. At $k=4$ a group of elderly women all with fracture history is detected, and at $k=$ 5 the group of elderly women with no fracture history is further divided into users and non-users of steroids. Up to this point the all-male cluster (cluster 1) and the young (cluster 4) cluster are intact. Table 2 summarises the characteristics of these 5 clusters.

Unadjusted incidence rates (and 95\% confidence intervals, CI) of fracture for these 5 clusters are shown in Figure 4. As expected the fracture risk of the cluster of elderly women with fracture history (cluster 5) is the highest (10.5/1000py), suggesting that those patients who have had a previous fracture are at highest risk of having a future one.
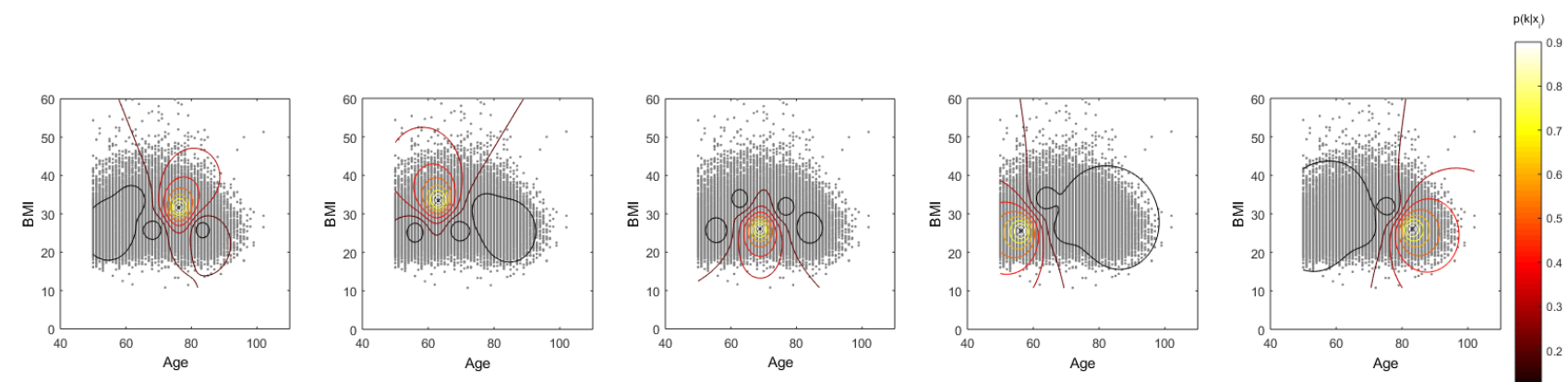

Figure 2. Contour plot of the distribution of cluster membership probabilityp $\left(j \mid x_{i}\right)$, along with cluster centroid (black cross). Each sub-plot represents the contour for one of 5 clusters. Also shown (gray dots) is the distribution of body mass index (y-axis) against age (x-axis) for all patients in the dataset.
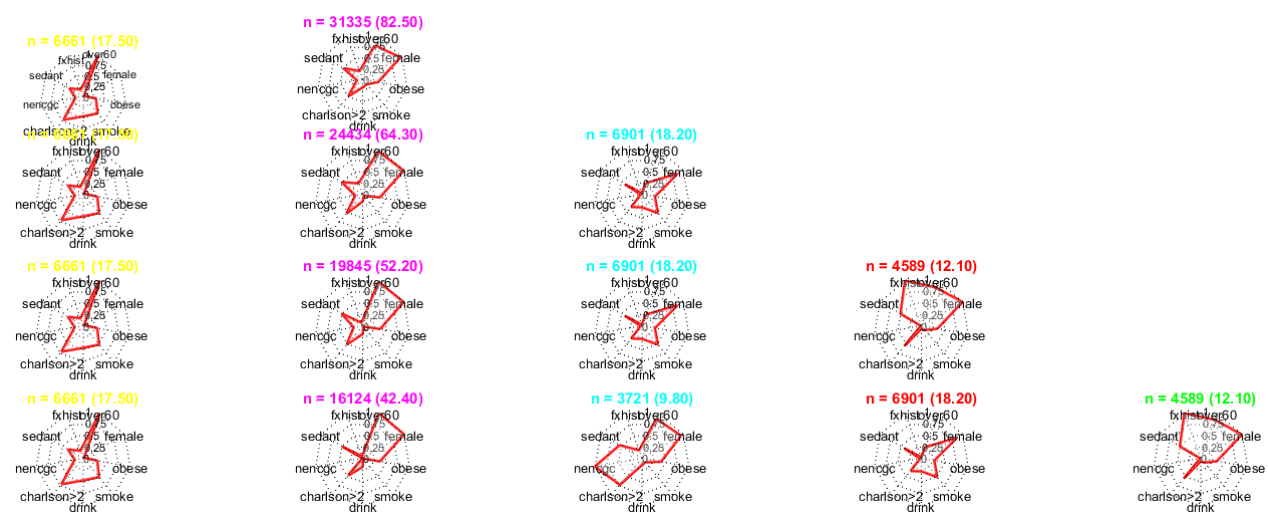

Figure 3: Distribution of the risk factors within a cluster obtained using hierarchical clustering. A feature can take values between 0 and 1 where e.g. female $=0$ indicates all subjects in this cluster are male, and female $=1$ indicates all subjects are female. The clusters corresponding to $k=2, k=3, k=4$ and $k=5$ are shown in panels from top to bottom, respectively. The number (n) and percentage (\%) of patients in each cluster is shown. 


\begin{tabular}{|l|r|r|r|r|r|}
\hline & \multicolumn{5}{|c|}{ Prevalence } \\
\hline Feature & Cluster 1 & Cluster 2 & Cluster 3 & Cluster 4 & Cluster 5 \\
\hline Elderly & 1.00 & 1.00 & 0.88 & 0.25 & 0.86 \\
\hline Female & 0.01 & 1.00 & 0.94 & 0.86 & 0.99 \\
\hline Obese & 0.29 & 0.36 & 0.40 & 0.27 & 0.34 \\
\hline Smoker & 0.51 & 0 & 0.11 & 0.51 & 0.12 \\
\hline Drinker & 0.46 & 0.18 & 0.14 & 0.27 & 0.06 \\
\hline $\begin{array}{l}\text { Comorbi } \\
\text { d }\end{array}$ & 0.71 & 0.47 & 0.72 & 0.35 & 0.55 \\
\hline $\begin{array}{l}\text { Steroid } \\
\text { user }\end{array}$ & 0.18 & 0 & 1.00 & 0.02 & 0.01 \\
\hline $\begin{array}{l}\text { Sedative } \\
\text { user }\end{array}$ & 0.37 & 0.49 & 0.54 & 0.42 & 0.50 \\
\hline $\begin{array}{l}\text { Fracture } \\
\text { history }\end{array}$ & 0.16 & 0.04 & 0.17 & 0.02 & 1.00 \\
\hline
\end{tabular}

Table 2 Prevalence of features in each of 5 clusters detected using hierarchical clustering, where minimum and maximum prevalence can be 0 and 1 .

Interestingly, the fracture risk of the low-risk cluster (cluster 4) is lower (1.5/1000py) even than that of the general source population (2.23/1000py) [12]. This corresponds to the cluster with no fracture history, younger people with mild comorbidity and therefore seems plausible.

Further insight may be gained by considering the distribution of bone mineral density (measured by T-score) - a clinically validated and widely used surrogate for fracture risk or bone strength (where a low $\mathrm{T}$-score indicates poor bone strength) - for the clusters (Figure 5). While the median hip Tscore for cluster $4(-1.6)$ is higher than that of cluster $5(-2.2)$, their median spine T-score is the lowest $(-2.7)$ among all clusters (where median spine $\mathrm{T}$-score for cluster $5=-2.1$ ). The finding that despite a "healthy" hip T-score, their spine bone mineral density is low enough to be osteoporotic, could form the basis of an explanation for why this seemingly healthy cluster is on AOD use. This demonstrates how cluster analysis can help to develop and characterise drug user profiles.

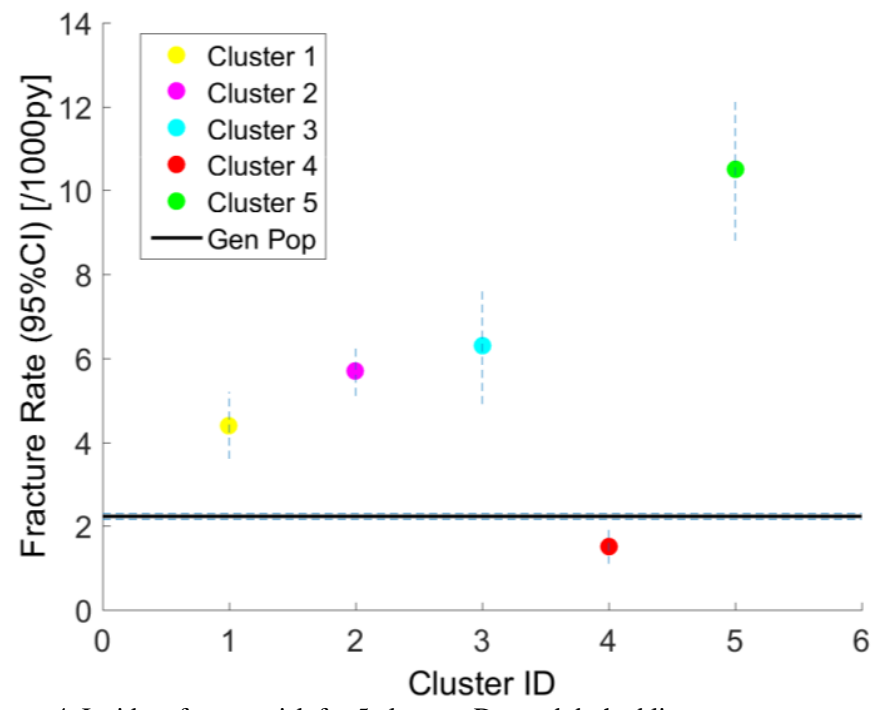

Figure 4. Incident fracture risk for 5 clusters. Dot and dashed line represent averege and $\pm 95 \%$ confidence intervals respectively. Bold black baseline represents population average.

\section{DISCUSSION AND CONCLUSION}

Internal evaluation exposed the difficulty in deriving an optimal number of clusters. It highlights that learning patterns from real-world data with highly complex and overlapped structures may require examination of clusters using external characteristics. Examining the bone mineral density and fracture risks for the detected groups, for instance, added to their plausibility.

These results should be interpreted in view of the fact that our dataset is complete-case i.e. these clusters only represent the sub-population of AOD users who reported their data in full. Similarly the BMD results are based on only those patients whose BMD was recorded. Further, one constraint when performing unsupervised learning or mining of electronic medical record data is that often clinical variables are recorded in a dichotomised fashion e.g. smoking status "yes" or "no", which limits the choice of methods that can be used for cluster analysis.

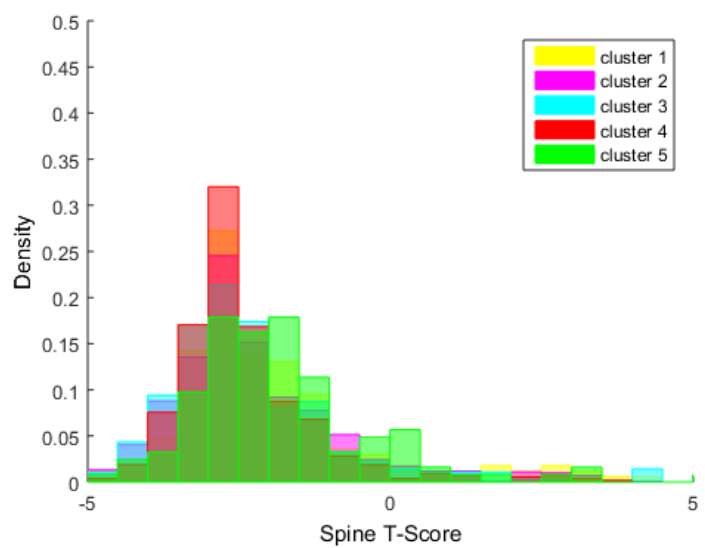

Figure 5. Bone mineral density for 5 clusters. 
Finally we were able to characterize clusters using only BMD and fracture risk because these data were available. Data on healthcare utilisation costs, private or public practice, and socio-economic status could further improve the characterisation of the clusters observed and lend credence to the clustering solution. In future work, we are in the process of extending our work to include novel unsupervised learning methods including deep learning which has the potential of performing selection of relevant clinical features in addition to cluster detection.

\section{ACKNOWLEDGMENT}

This work was supported by the NIHR Biomedical Research Centre, Oxford. DPA receives funding from the National Institute of Health Research (NIHR) in the form of a Clinician Scientist award (CS-2013-13-012).

\section{REFERENCES}

1. Kongkaew, C., P.R. Noyce, and D.M. Ashcroft, Hospital admissions associated with adverse drug reactions: a systematic review of prospective observational studies. Annals of Pharmacotherapy, 2008. 42(7-8): p. 1017-1025.

2. Multimorbidity: clinical assessment and management, $\mathrm{N}$. guideline, Editor., NICE guideline.

3. Jensen, P.B., L.J. Jensen, and S. Brunak, Mining electronic health records: towards better research applications and clinical care. Nature Reviews Genetics, 2012. 13(6): p. 395-405.
4. Wang, X., et al., Active computerized pharmacovigilance using natural language processing, statistics, and electronic health records: a feasibility study. Journal of the American Medical Informatics Association, 2009. 16(3): p. 328-337.

5. Bolíbar, B., et al., SIDIAP database: electronic clinical records in Primary Care as a source of information for epidemiologic research $\mid$ Base de datos SIDIAP: la historia clínica informatizada de Atención Primaria como fuente de información para la investigación epidemiológica. Medicina clinica, 2012.

6. Banerjee, A. and R.N. Dave. Validating clusters using the Hopkins statistic. in Fuzzy systems, 2004. Proceedings. 2004 IEEE international conference on. 2004. IEEE.

7. Aggarwal, C.C. and C.K. Reddy, Data clustering: algorithms and applications. 2013: CRC press.

8. MacQueen, J. Some methods for classification and analysis of multivariate observations. in Proceedings of the fifth Berkeley symposium on mathematical statistics and probability. 1967. Oakland, CA, USA.

9. Johnson, S.C., Hierarchical clustering schemes. Psychometrika, 1967. 32(3): p. 241-254.

10. Bishop, C., Pattern Recognition and Machine Learning (Information Science and Statistics), 1st edn. 2006. corr. 2nd printing edn. Springer, New York, 2007.

11. Bezdek, J.C., Pattern recognition with fuzzy objective function algorithms. 2013: Springer Science \& Business Media.

12. Pagès-Castellà, A., et al., Burden of osteoporotic fractures in primary health care in Catalonia (Spain): a population-based study. BMC musculoskeletal disorders, 2012. 13(1): p. 79.

13. Khalid, S., A. Judge, and R. Pinedo-Villanueva, An unsupervised learning model for pattern recognition in routinely collected healthcare data. 2018. 